\title{
Vestibular Neuritis in Patients Among Different Age Groups: Clinical Features and Outcomes
}

\author{
Tao Yan, MD, PhD ${ }^{1,2}$ Fangru Zong, MD ${ }^{3}$ Xiao Han, MD ${ }^{1,2}$ Xiaojing Wang, MD ${ }^{1,2}$ Qiuhong Li, MD ${ }^{1,2}$ \\ Ruru Qiao, MD ${ }^{1,2}$ Hanbing Zhang, MD, PhD ${ }^{1,2}$ \\ ${ }^{1}$ Department of Otorhinolaryngology, Qilu Hospital of Shandong \\ University, Jinan, People's Republic of China \\ 2 NHC Key Laboratory of Otorhinolaryngology, Shandong University, \\ Jinan, People's Republic of China \\ ${ }^{3}$ Department of Pediatrics, Qilu Hospital of Shandong University, \\ Jinan, People's Republic of China \\ J Am Acad Audiol 2020;31:629-635. \\ Address for correspondence Hanbing Zhang, MD, PhD, \\ Zhanghanbing1976@163.com
}

\begin{abstract}
Keywords

- vestibular neuritis

- clinical feature

- treatment

- recovery

- DHI

- HADS
\end{abstract}

Background Patients with vestibular neuritis (VN) displayed differential prognosis despite of the same treatment. Thus, identifying unique characteristics in different populations and creating individually customized treatments are necessary. However, studies about the clinical features according to different ages are scarce.

Purpose This article compares the differences in VN patients among different age groups.

Research Design A prospective study.

Study Sample A total of $70 \mathrm{VN}$ patients were enrolled in the present study.

Intervention All the patients started vestibular rehabilitation at the time of initial presentation to our clinic. They were followed up at 1-month intervals using the questionnaire until 4 months.

Data Collection and Analysis Patients' clinical data including clinical presentation, vestibular testing results, treatment, and recovery was collected and analyzed with Duncan's multiple range test, the sign test, and the Kruskal-Wallis test using SPSS18.0. Results The mean age of the 70 patients was $47.2 \pm 17.1$, ranging from 10 to 76 years old. The sex ratios (male:female) were 3.5 in the adolescent group, 0.643 in the young adult group, 1.375 in the middle-aged group, and 0.583 in the senior group. The prevalence of hypertension and diabetes mellitus showed a significantly increasing trend from young adults to the seniors $(p<0.05)$. The caloric response was statistically worse in the senior group than the other groups $(p<0.05)$. The abnormal rates for video head impulse test, vestibular-evoked myogenic potential, and vestibular autorotation test did not differ significantly in different age groups. A significant difference between prerehabilitation and postrehabilitation total Dizziness Handicap Inventory (DHI) scores was identified in all the groups $(p<0.05)$. The younger patients demonstrated a greater improvement than patients in the senior group, meanwhile adolescents improved the most $(p<0.05)$. Hospital Anxiety and Depression Scale (HADS) was the lowest in the adolescent group $(p<0.05)$. DHI score at acute stage was significantly correlated with $\operatorname{HADS}(r=0.597, p<0.05)$. received

October 31, 2019

accepted after revision

February 14, 2020

published online

October 9, 2020
(C) 2020. American Academy of

Audiology. All rights reserved. Thieme Medical Publishers, Inc., 333 Seventh Avenue, 18th Floor, New York, NY 10001, USA
DOI https://doi.org/ 10.1055/s-0040-1717067. ISSN 1050-0545. 
Conclusion The canal response was statistically better for younger patients compared with the elderly. The younger patients demonstrated a greater improvement than patients in the senior group, among whom adolescents improved the most, meanwhile psychological factors played a minor role in adolescents. Self-perceived disability-handicap positively correlated with anxiety and depression in all patients.

Vestibular neuritis (VN) is a disorder characterized by sudden vertigo with associated nausea, vomiting, and generalized imbalance. ${ }^{1}$ Thirty to $50 \%$ of the patients developed disabling chronic dizziness, which was significantly associated with reduced quality of life. ${ }^{2}$ The specific etiology of VN remains unclear, possible mechanisms include viral infection, vascular ischemic insults, autoimmune, or labyrinthitis. $^{3}$ Oral corticosteroids and vestibular rehabilitation have been shown efficacious in the treatment of acute vestibular loss. ${ }^{4,5}$ However, different populations displayed differential prognosis despite receiving the same treatment. ${ }^{5,6}$ Thus, identifying unique characteristics in different populations and creating individually customized treatments are necessary. However, studies about the clinical features according to different ages are scarce. In the study, we divided the patients in four groups according to ages: adolescent group, young adult group, middle-aged group, and senior group. We now report a prospective study to compare the differences of clinical features and outcomes in VN patients among different age groups.

\section{Methods}

\section{Patients}

The study was approved by the ethics committee in our hospital. A total of 70 consecutive patients diagnosed with acute unilateral VN were included initially from January 2017 to December 2018 at the department of otorhinolaryngology. The diagnosis of VN was based on: (1) a single sudden onset of sustained vertigo, (2) unidirectional spontaneous nystagmus that has horizontal components or combination of torsional components to the healthy side, (3) reduced caloric response (canal paresis $>25 \%$ ), and (4) absence of hearing loss and other neurologic signs. The exclusion criteria were (1) central lesions and (2) history of any other otologic disease.

\section{Vestibular Testing}

All the patients underwent pure-tone audiometry and a variable combination of objective vestibular tests, including the random amplitude saccade, smooth pursuit, optokinetic, and optokinetic after nystagmus. All testing was conducted in our clinical vestibular laboratory, department of otorhinolaryngology, by two licensed audiologists. Testing was performed at the time of initial patient presentation to our clinic.

Videonystagmography and binaural bithermal air caloric tests were performed using Micromedical equipment (GN Otometrics, Taastrup, Denmark). The maximum velocity of the slow phase components of nystagmus evoked in each ear was analyzed to identify unilateral weakness and directional preponderance as determined by Jongkees' formula. ${ }^{7}$ Canal paresis more than $25 \%$ was used as evidence of impaired unilateral vestibular nerve function.

Otolith function was measured using air-conducted sound (ACS) stimulation of cervical and ocular vestibular-evoked myogenic potentials (cVEMP, oVEMP) (EclipsVemp, Interacoustic, Assens, Denmark). ACS with a $500-\mathrm{Hz}$ short tone burst (rise $/$ fall time $=2 \mathrm{~ms}$; plateau time $=2 \mathrm{~ms}$ ) was used in a soundproof examination room. A stimulus level of 123.5-dB sound pressure level was used as the default starting intensity to check whether the subject's VEMPs could be elicited and to identify the waveforms. For the cVEMP test, active electrodes were placed on the upper half of the bilateral sternocleidomastoid muscles (SCMs), while reference and ground electrodes were placed on the suprasternal notch and forehead, respectively. Each subject was instructed to sit with the head rotated toward the ear not receiving the auditory stimulus. For the oVEMP test, the active electrode was placed $1 \mathrm{~cm}$ below the lower lid margin of each eye in line with the pupil. The reference electrode was placed $1 \mathrm{~cm}$ below the active electrode, and the ground electrode on the midline of the forehead. Each subject was asked to gaze upwards when hearing sound from the inserted earphone. The results were defined as abnormal in the absence of VEMP response or more than $40 \%$ of interaural amplitude difference ratio.

Video head impulse test (VHIT) was performed using the ICS Impulse system (GN Otometrics). In a brightly lit room, subjects sat on an office chair and were instructed to fix their eyes on a small dark target on the wall at $1.5 \mathrm{~m}$. Experienced audiologists performed brief, abrupt, and unpredictable head rotations on patients. At least 20 impulses with peak velocity ranges of 100 to 250/s were collected from each canal. Individual vestibulo-ocular reflex (VOR) gains were automatically calculated. A VHIT gain of $<0.8$ for the horizontal canals and $<0.7$ for the vertical canals as well as the occurrence of corrective saccades were considered pathologic.

Vestibular autorotation test (VAT) (Western Systems Research Inc., Pasadena, CA) was performed to assess vestibular function over a frequency range similar to head movement frequencies during functional activities. After cleaning with alcohol, subjects were fitted with five electrodes placed at the outer canthus of each eye, above and below the left eye in a line bisecting the middle of the pupil on primary gaze, and one placed slightly above the bridge of the nose. Subjects were instructed to move the head side to side in time with the tone of the sound made by the computer while keeping the eyes focused on the black dot. Target height was adjusted so that the 
relative position of the eyes in the orbit was always in the horizontal plane. It is recommended to allow the patient to practice at least twice before starting and recording the test procedure. Horizontal head movement is performed first followed by vertical movement. The gain, phase in horizontal and vertical direction together with asymmetry, is plotted against the different frequencies. Low horizontal and/or vertical gain accompanied by a delay in phase was considered as peripheral vestibular pathology.

\section{Questionnaire Measures}

To evaluate patients' subjective dizziness, the Chinese version of the Dizziness Handicap Inventory $(\mathrm{DHI})^{8}$ was used. A normalized score was used as patients in the acute stage were not able to answer all questions, for example, "Does walking down the aisle of a supermarket increase your problem?" The normalized score was calculated by dividing the total score by number of questions answered. The questionnaires were administered during patients' initial visits and then regularly followed up during vestibular rehabilitation.

The Chinese version of the Hospital Anxiety and Depression Scale (HADS) ${ }^{9}$ was used to measure state anxiety and depression. For each scale, scores ranged from 0 to 21 (high level of anxiety/depression). A cutoff score of 12 points was used to screen for general psychological distress.

\section{Vestibular Rehabilitation and Patient Follow-Up}

Vestibular rehabilitation was started at the time of initial patient presentation to our clinic. Rehabilitation program was conducted under the guidance of a qualified physiotherapist. A vestibular suppressant was stopped before rehabilitation in all the patients. The patients were instructed to rotate and move their head up and down while keeping their eyes fixed on a stationary target for 1 to 2 minutes three times per day. After being familiarized with the exercise, the patients were instructed to perform the same head movement with the eyes fixed on the target moving in the opposite direction. They were also given instructions regarding exercises to improve posture and gait, which were started after VOR exercise. Balance exercises comprised 30 repeated forward reaching exercises, 5 minutes of one leg standing, and 5 minutes of tandem standing. After maintaining this posture, they were directed to attempt to march in place. Further gait exercises comprised 10 minutes of continuous walking and 5 minutes of tandem walking. If above exercises could be performed without an issue, horizontal and vertical head movements were then added during the gait exercises. The physiotherapist adjusted the amount of exercise according to the patient's individual symptoms. Once the patients were familiar with the exercises, they were asked to repeat the exercise at least three times per day for 4 months at home.

The DHI questionnaire and VAT were performed to identify the recovery status of patients. The patients were followed up at 1-month intervals using the questionnaire and VAT until 4 months.

\section{Statistical Analysis}

Statistical analysis of the data was conducted with SPSS 18.0 (SPSS, Inc., Chicago, IL). The data were analyzed with Duncan's multiple range test, the sign test, and the KruskalWallis test. The relationship between level of anxiety/depression and subjective dizziness score was analyzed using multiple linear regression. Values are presented as the mean \pm standard deviation (SD). A p-value lower than 0.05 was regarded as statistically significant.

\section{Results}

The general complaints in our cohort include sudden onset of rotary vertigo, imbalance, nausea, vomiting, palpitation, and chest stuffiness. The mean age of the 70 patients was $47.2 \pm 17.1$, ranging from 10 to 76 years old. The patients were divided into four groups according to their ages (-Table 1). The sex ratios (male:female) were 3.5 in the adolescent group, 0.643 in the young adult group, 1.375 in the middle-aged group, and 0.583 in the senior group. The prevalence of hypertension was identified as $4.3 \%$ in the young adult group to $31.6 \%$ in the middle-aged group to $42.1 \%$ in the senior group, showing a significantly increasing sequence $(p<0.05 ;-$ Table 1$)$. The prevalence of diabetes mellitus showed a significantly increasing trend from young adults $(0 \%)$ to the seniors $(31.6 \%, p<0.05 ;-$ Table 1$)$. Patients were first evaluated in our department between 1 and 21 days from symptom onset (mean $10 \pm 11.6$ ).

Table 1 Patient demographics

\begin{tabular}{|l|l|l|l|l|}
\hline & Adolescent & Young adult & Middle-aged & Senior \\
\hline Age, y; mean \pm SD (range) & $16.6 \pm 5.25(10-29)$ & $38.35 \pm 5.04(30-45)$ & $56.12 \pm 5.32(46-64)$ & $68.38 \pm 3.36(65-76)$ \\
\hline Clients, $n$ & 9 & 23 & 19 & 19 \\
\hline Sex, $n(\%)$ & & & & \\
\hline Male & $7(77.8)$ & $9(39.1)$ & $11(57.9)$ & $7(36.8)$ \\
\hline Female & $2(22.2)$ & $14(60.9)$ & $8(42.1)$ & $12(63.2)$ \\
\hline Comorbidity, $n(\%)$ & & & $6(31.6)$ & $8(42.1)$ \\
\hline Hypertension & $0(0)$ & $1(4.3)$ & $2(10.5)$ & $6(31.6)$ \\
\hline Diabetes & $0(0)$ & $0(0)$ & & \\
\hline
\end{tabular}

Abbreviation: SD, standard deviation. 


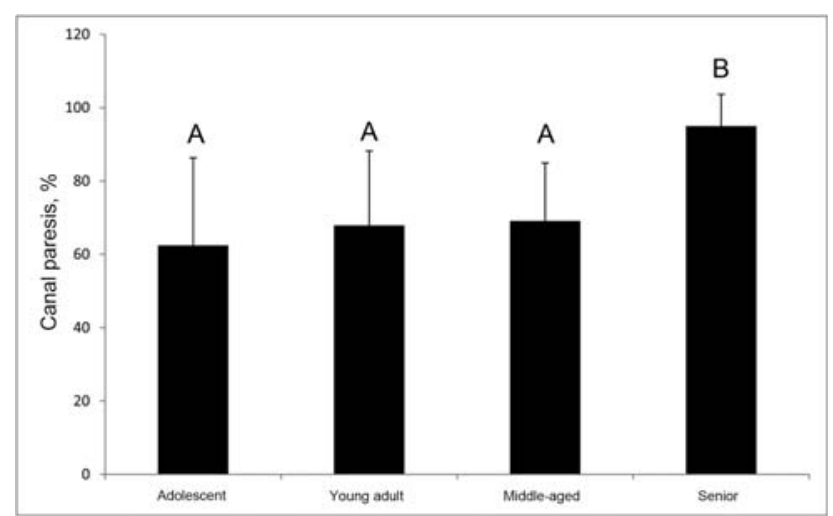

Fig. 1 Caloric paresis percentages in different age groups (mean \pm standard deviation [SD]). Percentages were calculated using the vestibular paresis formula by Jongkees et al, which indicates the extent of unilateral caloric paresis. Means with different letters are significantly different (sign test, $p<0.05$ ).

All the patients showed unilateral caloric weakness on a bithermal caloric test, whose mean (SD) canal paresis was 69.3 (20.8). When comparing the extent of vestibular paresis in different age groups, the canal response was statistically worse in the senior group than the other groups (-Fig. 1). The abnormal rates of horizontal plane of VHIT ran from $77.8 \%$ (adolescent), to $91.3 \%$ (young adult), to $94.7 \%$ (middleaged), to $100 \%$ (senior), showing an increasing trend with age, though not significant ( - Table 2 ). Similarly, as respect to the anterior plane and posterior plane, the abnormal rates increased with age, however, not significant (-Table 2 ). The abnormal rate of oVEMP was identified as $66.7 \%$ in the adolescent group, $73.9 \%$ in the young adult group, $89.5 \%$ in the middle-aged group, and $94.7 \%$ in the senior group $(p<0.05 ;-$ Table 2$)$. Meanwhile, the abnormal rate of cVEMP was $44.4 \%$ for the adolescents, $47.8 \%$ for the young adults, $47.4 \%$ for the middle-aged adults, and $57.9 \%$ for the seniors ( $p>0.05 ;-$ Table 2 ). The percentage of patients with low horizontal and/or vertical gain accompanied by a delay in phase tested by VAT did not differ significantly in different age groups (-Table 2). All patients had a normal audiogram.

DHI scores at the time of initial patient presentation to our clinic were comparable in the four groups. The sign test identified a significant difference between prerehabilitation and

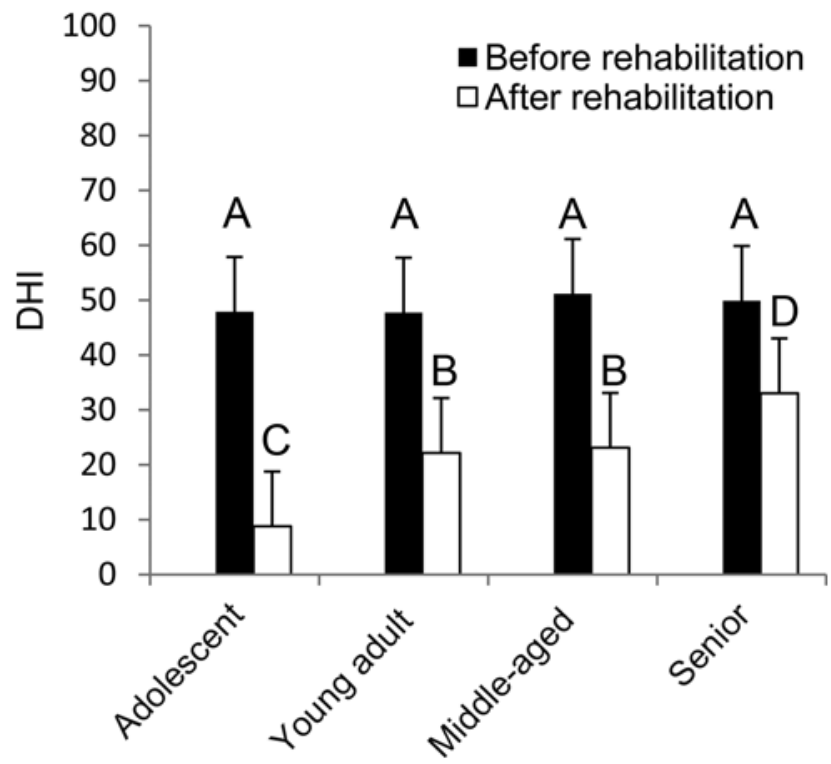

Fig. 2 Dizziness Handicap Inventory (DHI) scores before and after rehabilitation in different age groups (mean \pm standard deviation [SD]). Means with different letters are significantly different (sign test and Kruskal-Wallis test, $p<0.05$ ).

postrehabilitation total DHI scores in all the groups (-Fig. 2). The younger patients demonstrated a greater improvement than patients in the senior group. Interestingly, adolescents improved more than patients both in the young adult group and middle-aged group (-Fig. 2). There was no significant difference in the amount of change in DHI scores between the young adult group and the middle-aged group (-Fig. 2 ).

HADS was the lowest in the adolescent group compared with the older group, a significant difference was identified between the adolescent group and young adult group (-Fig. 3A). An age-dependent decrease in scores on the HADS was found in adults, though no significant differences revealed ( - Fig. 3A). Ten (44\%) patients were above the cutoff score for psychological disorders in the young adult group, the relative frequencies were $37 \%$ in the middle-aged group and $26 \%$ in the senior group. However, nobody demonstrated clinically relevant level of psychological distress in the adolescent group (-Fig. 3B). Interestingly, DHI score at acute stage

Table 2 Vestibular testing results

\begin{tabular}{|l|l|l|l|l|}
\hline & Adolescent & Young adult & Middle-aged & Senior \\
\hline Abnormal VHIT, $n(\%)$ & & & & \\
\hline Anterior plane & $6(66.7)$ & $19(82.6)$ & $17(89.5)$ & $18(94.7)$ \\
\hline Horizontal plane & $7(77.8)$ & $21(91.3)$ & $8(42.1)$ & $19(100)$ \\
\hline Posterior plane & $3(33.3)$ & & & $12(63.2)$ \\
\hline Abnormal VEMP, $n(\%)$ & & $17(73.9)$ & $17(89.5)$ & $9(47.4)$ \\
\hline oVEMP & $6(66.7)$ & $11(47.8)$ & $18(94.7)$ & $18(94.7)$ \\
\hline cVEMP & $4(44.4)$ & $22(95.7)$ & $11(57.9)$ \\
\hline Abnormal VAT, $n(\%)$ & $8(88.9)$ & $19(100)$ \\
\hline
\end{tabular}

Abbreviations: CVEMP, cervical vestibular-evoked myogenic potential; oVEMP, ocular vestibular-evoked myogenic potential; VAT, vestibular autorotation test; VHIT, video head impulse test. 

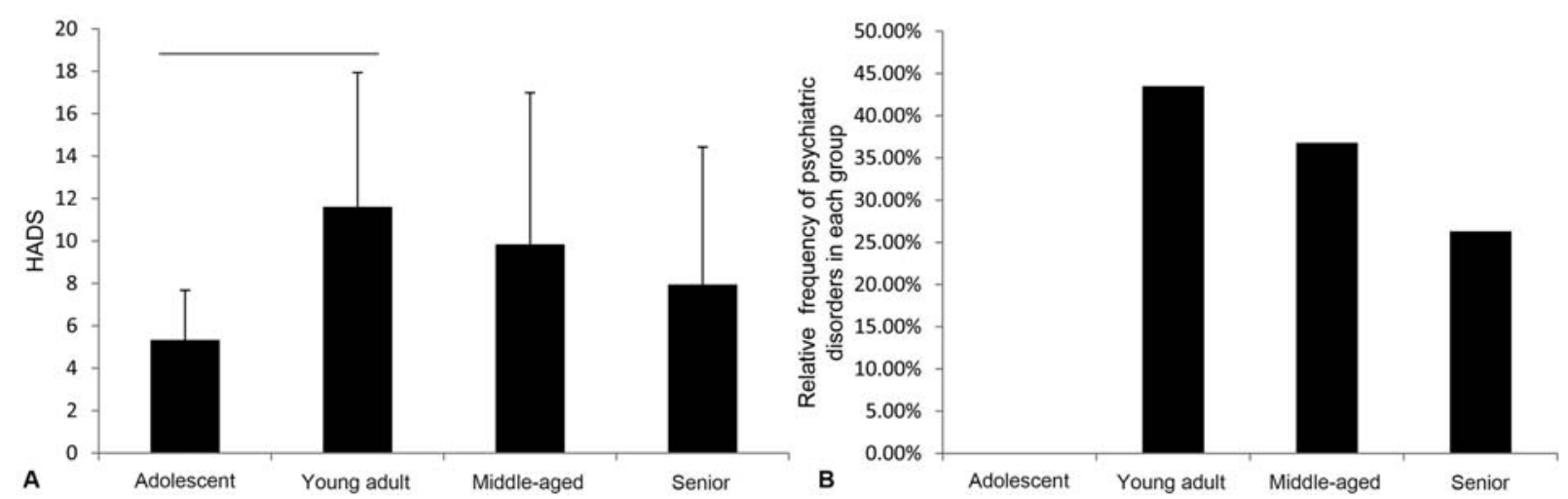

Fig. 3 (A) Hospital Anxiety and Depression Scale (HADS) in different age groups (mean \pm standard deviation [SD]). Line over bars indicates groups which were significantly different (Duncan's multiple range test, $p<0.05$ ). (B) The relative frequencies of psychiatric disorders in each group.

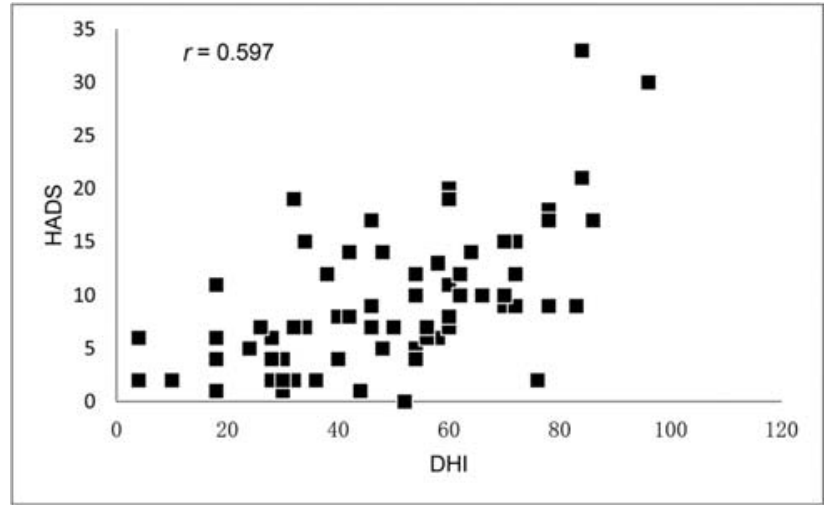

Fig. 4 Scatterplot showing the relationship between the Dizziness Handicap Inventory (DHI) scores and Hospital Anxiety and Depression Scale (HADS) scores in all the patients.

was significantly correlated with HADS across all age groups $(r=0.597, p<0.05)$ (-Fig. 4).

\section{Discussion}

Studies demonstrated VN to be the third most common cause of vertigo, making up approximately $7 \%$ of adult vertigo cases ${ }^{10}$ and $5 \%$ of cases of pediatric vertigo. ${ }^{11}$ In our study, the symptoms were similar between adolescents and adults. The majority of patients had been evaluated by multiple other physicians. However, none of the patients initially came to our department with a diagnosis of VN made prior to their visit, which implies the need for increased awareness of the features and assessment of VN among frontline doctors.

Vestibular function tests are essential for locational and qualitative diagnosis of VN. With the introduction of VHITs and VEMPs, it became evident that both vestibular nerve divisions could be affected. ${ }^{12}$ Meanwhile, atypical patterns involving only ampullary fibers or otolith afferents have been described. ${ }^{12}$ Studies suggested the extent of vestibular impairment was important in recovery of $\mathrm{VN} .^{13,14}$ Indeed, in our study, the canal response was statistically worse in the elderly, probably due to the loss of inner ear function from underlying pathology coupled with aging process, ${ }^{15}$ conse- quently they demonstrated a lesser improvement in DHI scores compared with younger patients. A recent study ${ }^{16}$ showed a significant correlation between age and VOR gain, the saccade profile of normal subjects on the horizontal VHIT. Moreover, both the cVEMP and oVEMP tests show an age-related deterioration in the corresponding neural functions. ${ }^{17,18}$ However, our study did not find significant difference in the abnormal rates for VHIT and VEMP in different age groups despite the increased abnormal trend with age observed. Further studies with larger VN populations to investigate VOR gain and saccade metrics in different age groups are warranted.

All the patients received a combination of medical management and rehabilitation exercise. Clinical recovery, as measured with DHI before and after treatment, demonstrated significant improvement among patients in all groups. When comparing adolescents with adults, there was significant difference in terms of DHI improvement. This means that the adolescent patients profited especially in mobility, autonomy, and vestibular balance. The results were in accordance with previous study, ${ }^{6}$ the striking difference in recovery was likely the result of more efficient vestibular compensation by children. Indeed, besides the regular rehabilitation exercise, children were more active than adults, ${ }^{19}$ showing a greater range of head positions related to sports, and other recreational activities. Moreover, boys, accounting for $77.8 \%$ in the adolescent group in our study, were more likely than girls to be observed in vigorous-intensity physical activity. ${ }^{20}$

According to our study, the psychiatric comorbidity was different among the four age groups (0, 44, 37, and 26\%). In comparison, a national survey of the U.S. general population reported the prevalence of mood disorders was $3.7 \%$ in youth ${ }^{21}$ and $6.6 \%$ in adults. ${ }^{22}$ Our data was in accord with the previous reports, ${ }^{23,24}$ psychiatric comorbidities, especially anxiety and depression, are more common in patients with vestibular disorders. The prevalence of anxiety and depression in adult patients was striking, suggesting that our findings warrant attention by health care providers. Indeed, it has been reported that psychiatric disorders can delay the recovery of imbalance and subjective dizziness after rehabilitation. ${ }^{4}$ In contrast to adults, none of the adolescent patients displayed psychiatric 
disorders. Accordingly, they showed dramatic improvement in DHI. Our data also demonstrated an age-dependent decrease in the portion of psychological diagnoses in the adult patients, though the differences were not statistically significant. Dietzek et al ${ }^{25}$ reported elderly patients suffering from dizziness scored significantly lower in HADS than the younger patients; moreover, the elderly improved 6 months after therapy especially in Mobility Inventory when alone and in Vertigo Severity Scale (vestibular-balance subscale). However, in our study, the elderly did not show greater improvement in DHI than other age groups. The inconsistency might be due to different patient populations. The extent of vestibular paresis, evidenced by statistically worse canal response in the elderly in our study, had a significant influence on the prognosis of static postural stability. ${ }^{26}$ Moreover, besides the lateral peripheral lesion, disequilibrium in the elderly often is multifactorial origin including multisensory deficits, as an increasing prevalence of comorbidities was noted from the young adult to the elderly in our study. Future studies with large sample size in selected populations are needed to clarify the controversy.

According to our study, the total scores on the DHI showed significant positive correlations with HADS in all patients, suggesting a strong relationship between psychological distress and dizziness handicap. ${ }^{24,27}$ Thus, besides the objective vestibular function test in the patients, it stresses the demand to apply DHI to evaluate the self-perceived handicap of balance system. If possible, every patient should get a psychological evaluation as well. Comprehensive early diagnosis allows more targeted therapies at the early phase of a vestibular injury.

There are several limitations to our study. First, our study was limited by a small sample size, especially the relatively small sample size in the adolescent group. Second, the efficacy of the treatments was influenced by the variability in the timing of our initial evaluations of patients. Third, it might be biased by the selection of patients showing compliance with follow-up. Despite these limitations, our data showed striking differences for VN patients among different age groups, and highlight that clinicians should look for anxiety/depression more aggressively in all patients with VN.

\section{Conclusion}

In conclusion, VN patients in different age groups showed different characteristics. The caloric response was statistically better for younger patients compared with the elderly. The younger patients demonstrated a greater improvement than patients in the senior group, among whom adolescents improved the most; meanwhile, psychological factors played a minor role in adolescents. Self-perceived disability-handicap positively correlated with anxiety and depression in all patients. In the future, age-related multimodal therapeutic interventions have the potential to prevent further chronification and induce persistent improvement.

\section{Conflict of Interest}

None.

\section{References}

1 Baloh RW. Clinical practice. Vestibular neuritis. N Engl J Med 2003;348(11):1027-1032

2 Halmagyi GM, Weber KP, Curthoys IS. Vestibular function after acute vestibular neuritis. Restor Neurol Neurosci 2010;28(01):37-46

3 Jeong SH, Kim HJ, Kim JS. Vestibular neuritis. Semin Neurol 2013; 33(03):185-194

4 Herdman SJ. Vestibular rehabilitation. Curr Opin Neurol 2013;26 (01):96-101

5 Strupp M, Zingler VC, Arbusow V, et al. Methylprednisolone, valacyclovir, or the combination for vestibular neuritis. N Engl J Med 2004;351(04):354-361

6 Brodsky JR, Cusick BA, Zhou G. Vestibular neuritis in children and adolescents: clinical features and recovery. Int J Pediatr Otorhinolaryngol 2016;83:104-108

7 Jongkees LB, Maas JP, Philipszoon AJ. Clinical nystagmography. A detailed study of electro-nystagmography in 341 patients with vertigo. Pract Otorhinolaryngol (Basel) 1962;24:65-93

8 Jacobson GP, Newman CW. The development of the Dizziness Handicap Inventory. Arch Otolaryngol Head Neck Surg 1990;116 (04):424-427

9 Zigmond AS, Snaith RP. The hospital anxiety and depression scale. Acta Psychiatr Scand 1983;67(06):361-370

10 Strupp M, Brandt T. Vestibular neuritis. Semin Neurol 2009;29 (05):509-519

11 Wiener-Vacher SR. Vestibular disorders in children. Int J Audiol 2008;47(09):578-583

12 Taylor RL, McGarvie LA, Reid N, Young AS, Halmagyi GM, Welgampola MS. Vestibular neuritis affects both superior and inferior vestibular nerves. Neurology 2016;87(16):1704-1712

13 Hwang K, Kim BG, Lee JD, Lee ES, Lee TK, Sung KB. The extent of vestibular impairment is important in recovery of canal paresis of patients with vestibular neuritis. Auris Nasus Larynx 2019;46 (01):24-26

14 Jeong J, Jung J, Lee JM, Suh MJ, Kwak SH, Kim SH. Effects of saccular function on recovery of subjective dizziness after vestibular rehabilitation. Otol Neurotol 2017;38(07):1017-1023

15 Jeng YJ, Young YH. Evolution of vestibular disorders in older adults: from young-old to middle-old to oldest-old. Geriatr Gerontol Int 2020;20(01):42-46

16 Jay DR, Cane D, Howe S. Age is a greater influence on small saccades than target size in normal subjects on the horizontal video head impulse test. Front Neurol 2019;10:328

17 Su HC, Huang TW, Young YH, Cheng PW. Aging effect on vestibular evoked myogenic potential. Otol Neurotol 2004;25(06):977-980

18 Tseng CL, Chou CH, Young YH. Aging effect on the ocular vestibular-evoked myogenic potentials. Otol Neurotol 2010;31(06): 959-963

19 Floyd MF, Spengler JO, Maddock JE, Gobster PH, Suau LJ. Parkbased physical activity in diverse communities of two U.S. cities. An observational study. Am J Prev Med 2008;34(04):299-305

20 Kaczynski AT, Stanis SA, Besenyi GM, Child S. Differences in youth and adult physical activity in park settings by sex and race/ ethnicity. Prev Chronic Dis 2013;10:E42

21 Kabra AT, Feustel PJ, Kogan BA. Screening for depression and anxiety in childhood neurogenic bladder dysfunction. J Pediatr Urol 2015;11(02):75.e1-75.e7

22 Kessler RC, Berglund P, Demler O, et al; National Comorbidity Survey Replication. The epidemiology of major depressive disorder: results from the National Comorbidity Survey Replication (NCS-R). JAMA 2003;289(23):3095-3105

23 Jacob RG, Furman JM. Psychiatric consequences of vestibular dysfunction. Curr Opin Neurol 2001;14(01):41-46

24 Piker EG, Kaylie DM, Garrison D, Tucci DL. Hospital anxiety and depression scale: factor structure, internal consistency and convergent validity in patients with dizziness. Audiol Neurotol 2015; 20(06):394-399 
25 Dietzek M, Finn S, Karvouniari P, et al. In older patients treated for dizziness and vertigo in multimodal rehabilitation somatic deficits prevail while anxiety plays a minor role compared to young and middle aged patients. Front Aging Neurosci 2018;10:345

26 Fujimoto C, Egami N, Kinoshita M, Sugasawa K, Yamasoba T, Iwasaki S. Postural stability in vestibular neuritis: age, disease duration, and residual vestibular function. Laryngoscope 2014; 124(04):974-979

27 Piker EG, Jacobson GP, McCaslin DL, Grantham SL. Psychological comorbidities and their relationship to self-reported handicap in samples of dizzy patients. J Am Acad Audiol 2008;19(04): $337-347$ 